\title{
Theory of a cylindrical Langmuir probe parallel to the magnetic field and its calibration with interferometry
}

M. Usoltceva, E. Faudot, J. Ledig, S. Devaux, S. Heuraux, G. V. Zadvitskiy, R. Ochoukov, J. Moritz, K. Crombé, and J.-M. Noterdaeme

Citation: Review of Scientific Instruments 89, 10J124 (2018); doi: 10.1063/1.5038666

View online: https://doi.org/10.1063/1.5038666

View Table of Contents: http://aip.scitation.org/toc/rsi/89/10

Published by the American Institute of Physics

\section{Articles you may be interested in}

Record indoor magnetic field of $1200 \mathrm{~T}$ generated by electromagnetic flux-compression Review of Scientific Instruments 89, 095106 (2018); 10.1063/1.5044557

Development of a triple probe array for mode conversion study of electron cyclotron wave Review of Scientific Instruments 89, 10J121 (2018); 10.1063/1.5038852

Invited Review Article: Multi-tip scanning tunneling microscopy: Experimental techniques and data analysis Review of Scientific Instruments 89, 101101 (2018); 10.1063/1.5042346

Characteristics of the SOL turbulence structure in the first experimental campaign on W7-X with limiter configuration

Physics of Plasmas 25, 072502 (2018); 10.1063/1.5033353

Dual laser holography for in situ measurement of plasma facing component erosion (invited)

Review of Scientific Instruments 89, $10 \mathrm{J123}$ (2018); 10.1063/1.5039628

High resolution magnetic field measurements in hydrogen and helium plasmas using active laser spectroscopy

Review of Scientific Instruments 89, 10D126 (2018); 10.1063/1.5039334

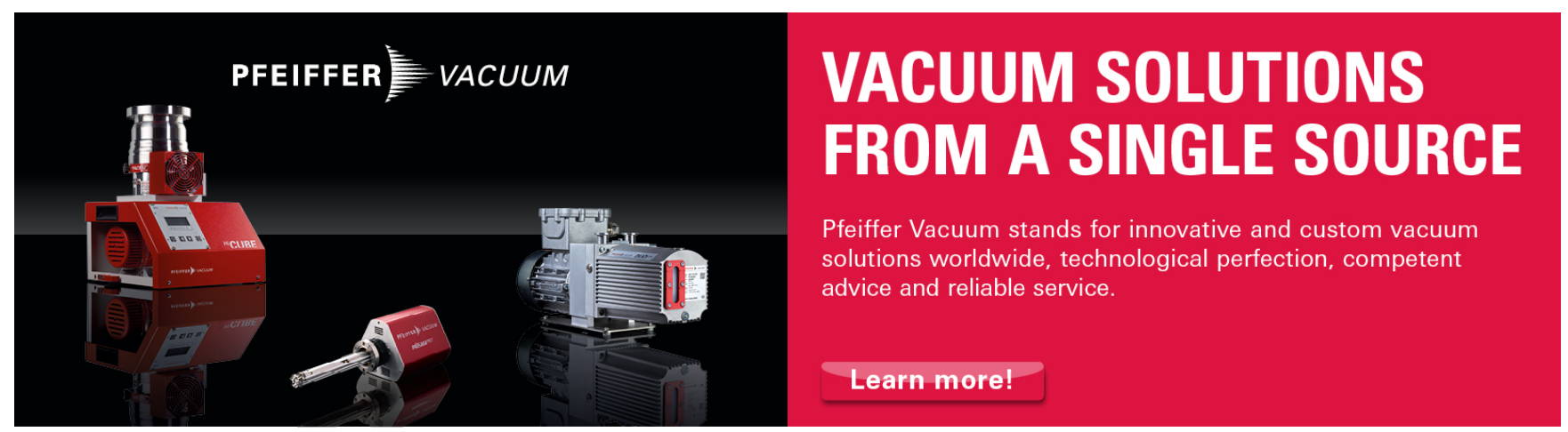




\title{
Theory of a cylindrical Langmuir probe parallel to the magnetic field and its calibration with interferometry
}

\author{
M. Usoltceva, ${ }^{1,2,3, a)}$ E. Faudot, ${ }^{2}$ J. Ledig, ${ }^{2}$ S. Devaux, ${ }^{2}$ S. Heuraux, ${ }^{2}$ G. V. Zadvitskiy, ${ }^{2,4}$ \\ R. Ochoukov, ${ }^{3}$ J. Moritz, ${ }^{2}$ K. Crombé, ${ }^{1,5}$ and J.-M. Noterdaeme ${ }^{1,3}$ \\ ${ }^{1}$ Ghent University, 9000 Ghent, Belgium \\ ${ }^{2}$ Université de Lorraine, 54506 Vandouvre-lès-Nancy, France \\ ${ }^{3}$ Max-Planck-Institut für Plasmaphysik, Boltzmannstr. 2, 85748 Garching, Germany \\ ${ }^{4}$ Institut für Grenzflächenverfahrenstechnik und Plasmatechnologie, Universität Stuttgart, 70569 Germany \\ ${ }^{5}$ LPP-ERM-KMS, Royal Military Academy, 1000 Brussels, Belgium
}

(Presented 17 April 2018; received 4 May 2018; accepted 4 July 2018; published online 15 October 2018)

\begin{abstract}
A theory for data interpretation is presented for a cylindrical Langmuir probe in plasma parallel to the magnetic field direction. The theory is tested in a linear low-temperature plasma device Aline, in a capacitive radio-frequency (RF) discharge. The probe is placed on a 3D manipulator, and a position scan is performed. To exclude strong RF perturbations, the probe is RF compensated. Using the theory, electron densities are obtained from the current at the plasma potential, where no sheath is present. Results are calibrated by line-integrated density measurements of a $26.5 \mathrm{GHz}$ microwave interferometer. Reasonable agreement is observed for probe and interferometer measurements. Furthermore, preceding, more general probe theory is compared to the one developed in the current work and the application limits are discussed. https://doi.org/10.1063/1.5038666
\end{abstract}

\section{INTRODUCTION}

Specific particle transport in the presence of magnetic field (B) changes the shape of a Langmuir probe IV curve such that the conventional methods of data interpretation become unsuitable, $, 1,2$ especially for a parallel cylindrical probe. Very few attempts ${ }^{1-3}$ exist on providing a correct theory for density (n) evaluation with such a probe. Of those, the Laframboise and Rubinstein calculations ${ }^{2,3}$ are hardly applicable in practice ${ }^{1}$ and our previous theory ${ }^{1}$ contains simplifying assumptions that restrict validity limits.

In this paper, we develop an accurate theory describing electron collection by a cylindrical Langmuir probe biased to plasma potential $\left(\mathrm{V}_{\mathrm{pl}}\right)$ (to avoid sheath) and oriented parallel to the magnetic field. This model is an advancement of our previous work, ${ }^{1}$ and it gives an exact formula connecting plasma density and collected current at $\mathrm{V}_{\mathrm{pl}}$. Only the case of a parallel probe is considered since for a probe at an arbitrary angle to $\mathrm{B}$ an exact analytical solution would be excessively complex. At the end, the applicability limits of the approximate theory ${ }^{1}$ are revised.

Application of the new theory to experimental data on Aline (A LINear Experiment ${ }^{4,5}$ ) allows us to draw spatial density profiles, as well as parameter scans. Results are validated with interferometry. A full scan of density as a function of B and the input power is performed for the first time on Aline in magnetized plasma.

\footnotetext{
Note: Paper published as part of the Proceedings of the 22nd Topical Conference on High-Temperature Plasma Diagnostics, San Diego, California, April 2018.

a) Author to whom correspondence should be addressed: mariia.usoltceva@ ipp.mpg.de
}

\section{THEORY FOR A PROBE PARALLEL TO B}

The basic idea behind the model presented here is the same as in the previous work: ${ }^{1}$ separation of different contributions to the collected current by dividing the electrons into groups according to their speed. The current for each contribution is calculated as a product of charge $e$, flux $\Gamma$, and collecting area $S$,

$$
I_{e}=e \Gamma S
$$

In contrast to the previous theory, we consider not only the flux to be dependent on the particle speed but the collecting area as well.

We adapt the cylindrical coordinate system and examine the particle flux through a plane perpendicular to the probe axis (z axis). The collecting area $S$ in (1) is an area in this plane which a guiding center of a rotating particle should cross for a particle to be collected. Due to the rotational symmetry, no dependency on the azimuth $\varphi$ is present. We divide all electrons into 4 fractions:

1. $v_{r} \leq v_{\lim r}, v_{z} \leq v_{\lim z}$;

2. $v_{r} \geq v_{\lim r}, v_{z} \leq v_{\lim z}$

3. $v_{r} \leq v_{\lim r}, v_{z} \geq v_{\lim z}$;

4. $v_{r} \geq v_{\lim r}, v_{z} \geq v_{\lim z}$,

where $v_{\lim r}=\frac{e B r_{p r}}{m}$ is the speed of an electron with a Larmor radius equal to the probe radius $r_{c}=r_{p r}$ and $v_{\lim z}=\frac{e B L_{p r}}{2 \pi m}$ is the speed of an electron that completes one full rotation $\Delta \varphi=2 \pi$ during the same time as it travels the probe length $L_{p r}$ in the $\mathrm{z}$ direction $\left(\frac{2 \pi}{\omega_{c}}=\frac{L_{p r}}{v_{\lim z}}\right.$, where $\omega_{c}=e B / m$ is the electron cyclotron frequency). For experimental applications, it would be correct to impose an upper limit for the radial speed as the 
value when the Larmor radius is equal to the vacuum chamber radius. This is a minor correction, and we omit it for the current theoretical formulation.

For each fraction of particles, the current is calculated using the same formula but with its own limits and its own collecting area (with Maxwellian velocity distribution),

$$
\begin{aligned}
I= & \operatorname{en}\left(\frac{m_{e}}{2 \pi k T_{e}}\right)^{\frac{3}{2}} \\
& \times \int_{v_{z_{1}}}^{v_{z_{2}}} \int_{0}^{2 \pi} \int_{v_{r_{1}}}^{v_{r_{2}}} S\left(v_{r}, v_{z}\right) v_{z} v_{r} \exp \left(-\frac{m v^{2}}{2 k T_{e}}\right) d v_{r} d \varphi d v_{z} .
\end{aligned}
$$

The assumptions and results for each of them are the following.

(1) These particles are "slow" in the $\mathrm{z}$ direction; they complete a full $2 \pi$ rotation before reaching the end of the probe. If the perpendicular projection of a particle trajectory intersects the probe projection in at least one point [Figs. 1(a) and 1(b)], a particle is for sure collected.

With $h$ being the distance between the probe center and the guiding center of a particle trajectory, the collecting area is found as

$$
S_{1}=2 \pi \int_{0}^{r_{p r}+r_{c}} h d h=\pi\left(r_{p r}+r_{c}\right)^{2} .
$$

Then the integration of (2) gives

$$
\begin{aligned}
I_{1}= & e \Gamma_{e}\left(1-e^{-\frac{v_{\lim z}^{2}}{a}}\right)\left[1-4 e^{-\frac{\beta^{2}}{2}}+\frac{2}{\beta^{2}}\left(1-e^{-\frac{\beta^{2}}{2}}\right)\right. \\
& \left.+\frac{\sqrt{2 \pi}}{\beta} \operatorname{erf}\left(\frac{\beta}{\sqrt{2}}\right)\right] \pi r_{p r}^{2},
\end{aligned}
$$

where $\beta=\frac{r_{p r}}{r_{c e}}=\frac{r_{p r} e B}{\sqrt{m_{e} k T_{e}}}$ is the nondimensional magnetic field strength ${ }^{2}$ and $\Gamma_{e}=n\left(\frac{k T_{e}}{2 \pi m_{e}}\right)^{1 / 2}$ is the random thermal flux.

(2) The same assumption is valid for the second fraction of electrons, except that the integration for $h$ is performed in different limits [see Figs. 1(c) and 1(d)],

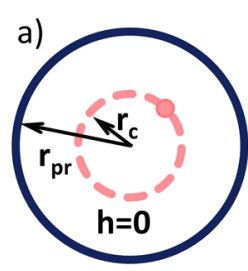

c)

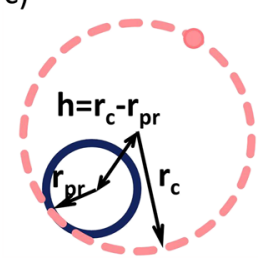

b)

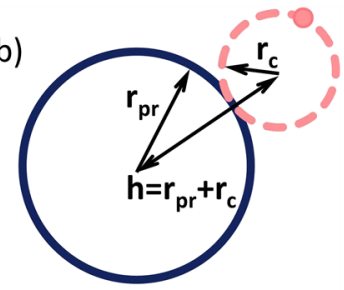

d)

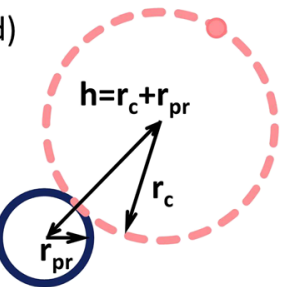

FIG. 1. Limiting cases for a particle to be collected: [(a) and (b)] for $r_{c}<r_{p r}$ and [(c) and (d)] for $r_{c}>r_{p r}$. A dark circle represents the probe projection, and a bright dashed circle represents the electron trajectory projection.

$$
\begin{gathered}
S_{2}=2 \pi \int_{r_{c}-r_{p r}}^{r_{c}+r_{p r}} h d h=4 \pi r_{p r} r_{c}, \\
I_{2}=e \Gamma_{e}\left(1-e^{-\frac{v_{\lim z}^{2}}{a}}\right)\left[4 e^{-\frac{\beta^{2}}{2}}+2 \frac{\sqrt{2 \pi}}{\beta} \operatorname{erfc}\left(\frac{\beta}{\sqrt{2}}\right)\right] \pi r_{p r}^{2} .
\end{gathered}
$$

(3) A particle that is "fast" in the $\mathrm{z}$ direction might never touch the probe because it does not complete a full turn on $L_{p r}$. This case should be approached probabilistically.

There are two sectors of the trajectory projection which correspond to the electron successful collection (Fig. 2). If a particle is in the sector $2 \alpha$ that belongs to the intersection part, it hits the probe perpendicular surface. From the law of cosines, $a=\arccos \left(\frac{r_{c}^{2}+h^{2}-r_{p r}^{2}}{2 r_{c} h}\right)$. The corresponding probability is $P_{\perp}=\frac{2 \alpha}{2 \pi}$. If a particle is in the sector $\gamma=\omega_{c} \frac{L_{p r}}{v_{z}}$, on the length $L_{p r}$ in the z direction, it completes a part of a full turn that is enough to reach the probe. So, a particle hits the parallel probe surface with $P_{\|}=\frac{\gamma}{2 \pi}$. Instead of $S$, now we need to use the product of $S$ and the full probability $P=P_{\perp}+P_{\|}$,

$$
S P=2 \pi \int_{h_{1}}^{h_{2}} \frac{2 \alpha+\gamma}{2 \pi} h d h=S P_{\perp}+2 \pi \gamma \int_{h_{1}}^{h_{2}} h d h,
$$

$$
\begin{aligned}
S P_{\perp}= & \left.h^{2} \arccos \left(\frac{r_{c}^{2}+h^{2}-r_{p r}^{2}}{2 r_{c} h}\right)\right]_{h_{1}}^{h_{2}} \\
& \left.-\frac{1}{2} \sqrt{2 h^{2}\left(r_{c}^{2}+r_{p r}^{2}\right)-h^{4}-\left(r_{c}^{2}-r_{p r}^{2}\right)^{2}}\right]_{h_{1}}^{h_{2}} \\
& -\left.r_{p r}^{2} \arctan \left(\frac{r_{c}^{2}-h^{2}+r_{p r}^{2}}{\sqrt{2 h^{2}\left(r_{c}^{2}+r_{p r}^{2}\right)-h^{4}-\left(r_{c}^{2}-r_{p r}^{2}\right)^{2}}}\right)\right|_{h_{1}} ^{h_{2}}
\end{aligned}
$$

With the limits $h_{1}=0$ and $h_{2}=r_{p r}+r_{c}$, we obtain

$$
S P_{3}=\pi r_{p r}^{2}+\frac{\omega_{c} L_{p r}}{2 \pi v_{z}} \pi\left(r_{p r}+r_{c}\right)^{2},
$$

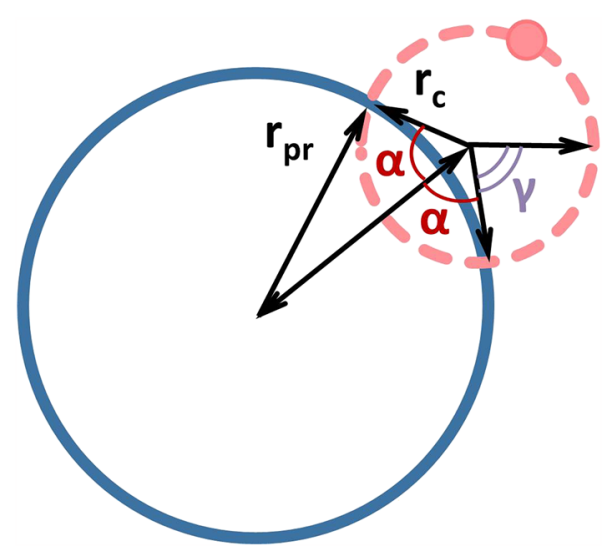

FIG. 2. Example of an intersection illustrating sectors $2 \alpha$ and $\gamma$. 


$$
\begin{aligned}
I_{3}= & e \Gamma_{e}\left(e^{-\frac{v_{\lim z}^{2}}{a}}\left(1-e^{-\frac{\beta^{2}}{2}}\right) \pi r_{p r}^{2}+\left[1-4 e^{-\frac{\beta^{2}}{2}}\right.\right. \\
& \left.+\frac{2}{\beta^{2}}\left(1-e^{-\frac{\beta^{2}}{2}}\right)+\frac{\sqrt{2 \pi}}{\beta} \operatorname{erf}\left(\frac{\beta}{\sqrt{2}}\right)\right] \\
& \left.\times \sqrt{\frac{\pi}{2}} \frac{\beta}{2} \operatorname{erfc}\left(\frac{v_{\lim z}}{\sqrt{a}}\right) r_{p r} L_{p r}\right) .
\end{aligned}
$$

(4) Like the previous calculation, but with other limits for $h$, for the 4th fraction, we get

$$
\begin{gathered}
S P_{4}=2 \pi \int_{r_{c}-r_{p r}}^{r_{c}+r_{p r}} \frac{2 \alpha+\gamma}{2 \pi} h d h=\pi r_{p r}^{2}+\frac{\omega_{c} L_{p r}}{2 \pi v_{z}} 4 \pi r_{p r} r_{c} \\
I_{4}=e \Gamma_{e}\left(e^{-\frac{v_{\lim z}^{2}}{a}} e^{-\frac{\beta^{2}}{2}} \pi r_{p r}^{2}+2\left[\sqrt{\frac{\pi}{2}} 2 \beta e^{-\frac{\beta^{2}}{2}}\right.\right. \\
\left.\left.+\pi \operatorname{erfc}\left(\frac{\beta}{\sqrt{2}}\right)\right] \operatorname{erfc}\left(\frac{v_{\lim z}}{\sqrt{a}}\right) r_{p r} L_{p r}\right) .
\end{gathered}
$$

The second term in $I_{4}$ has an additional factor of 2 to account for the particle collection from the opposite direction of $\mathrm{z}$. It is not done for the first term, as well as for all other current contributions, because the flow of the electrons is limited at the back of the probe tip by the probe body and the manipulator. Only particles with big $r_{c}$ and big $v_{z}$ are able to come from the back side.

The effective collecting area ${ }^{1}$ is the full collected current divided by $e \Gamma_{e}$,

$$
\begin{aligned}
S_{\text {eff }}= & \frac{I_{1}+I_{2}+I_{3}+I_{4}}{e \Gamma_{e}}=\left(e^{-\frac{v_{\lim z}^{2}}{a}}+\left[1-e^{-\frac{v^{2}}{a} z}\right]\right. \\
& \left.\times\left[1+\frac{2}{\beta^{2}}\left(1-e^{-\frac{\beta^{2}}{2}}\right)+\frac{\sqrt{2 \pi}}{\beta}\left(1+\operatorname{erfc}\left(\frac{\beta}{\sqrt{2}}\right)\right)\right]\right) \pi r_{p r}^{2} \\
& +\left[1+\operatorname{erfc}\left(\frac{\beta}{\sqrt{2}}\right)+\frac{1}{\sqrt{2 \pi} \beta}\left(\frac{\beta^{2}}{2}+2 \beta^{2} e^{-\frac{\beta^{2}}{2}}+1-e^{-\frac{\beta^{2}}{2}}\right)\right] \\
& \times \operatorname{erfc}\left(\frac{v_{\lim z}}{\sqrt{a}}\right) \pi r_{p r} L_{p r} .
\end{aligned}
$$

The expression is rather complicated, but it is important to see here that for $\beta \rightarrow \infty$ it converges to $S_{\perp}=\pi r_{p r}^{2}$ and for $\beta \rightarrow 0$ to $S_{p r}=2 \pi r_{p r} L_{p r}+\pi r_{p r}^{2}$.

\section{APPLICATION TO EXPERIMENTAL DATA}

Theory validation has been performed using data from the linear plasma device Aline. The experimental conditions were the same as in our previous paper: ${ }^{1} 25 \mathrm{MHz}$ capacitive radio-frequency (RF) discharge, magnetic fields of 0.0024-0.1 T, helium, and $1 \mathrm{~Pa}$. We have performed a scan of a probe position in the direction perpendicular to $\mathrm{B}$ ( $\mathrm{x}$ from $-33 \mathrm{~mm}$ to $+33 \mathrm{~mm}$ ) at a constant height $\mathrm{y}=36 \mathrm{~mm}$ above the antenna and at $\mathrm{z}=0 \mathrm{~mm}$ along $\mathrm{B}$, i.e., directly above the antenna center. In this region, densities are expected to be higher than at the position used before. ${ }^{1}$ Only one cylindrical probe tip of $\mathrm{r}_{\mathrm{pr}}=75 \mu \mathrm{m}, \mathrm{L}_{\mathrm{pr}}=1 \mathrm{~cm}$ was used, and it was accurately aligned with $\mathrm{B}$.

IV curve analysis, namely, plasma potential and electron temperature calculation, has been performed using accurate techniques described in our previous work. ${ }^{1}$ Densities obtained from the Langmuir probe are averaged along $\mathrm{x}$ and compared to line-integrated density measured by a $26.5 \mathrm{GHz}$ microwave interferometer MWI 2650 from Miwitron which was chosen because it suits our density range (Fig. 3). Interferometer, used only as a supportive diagnostic for probe calibration, was installed specifically for these tests on a temporary mounting, and mechanical shaking during experiments led to reduced accuracy. The lowest possible interferometry signal to measure was $\sim 1 \times 10^{16} \mathrm{~m}^{-3}$ in our experiments. Above this level, errors reached $\pm 1 \times 10^{16} \mathrm{~m}^{-3}$.

Possible sources of errors in the Langmuir probe results are as follows:

(a) RF oscillation of the potential, leading to a shift in IV characteristics. They are eliminated by the compensation circuit of our probe.

(b) Inaccurate $\mathrm{V}_{\mathrm{pl}}$ evaluation, leading to wrong current values. Using the intersection method of $\mathrm{V}_{\mathrm{pl}}$ calculation, ${ }^{1}$ we make sure that this issue is only present for very low $\mathrm{B}$, less than $0.005 \mathrm{~T}$ in our experiments.

(c) Uncertainties in temperature estimation affecting $n$ directly as $1 / \sqrt{T}$, as well as through the effective area. This should not give significant deviations when $T$ is determined self-consistently ${ }^{1}$ with $n$.

An additional uncertainty in the interferometer density values comes from the fact that the line-integrated signal is divided by a plasma length which is not exactly defined. For all points, it was divided by $20 \mathrm{~cm}$, which is fairly realistic for low plasma magnetization and overestimated (up to 2 times) for the higher B. The profile shape variation can be seen even on a small profile part that is accessible for the probe, nearly flat for the $0 \mathrm{~T}$ field and with a defined peak for greater field values (Fig. 4).

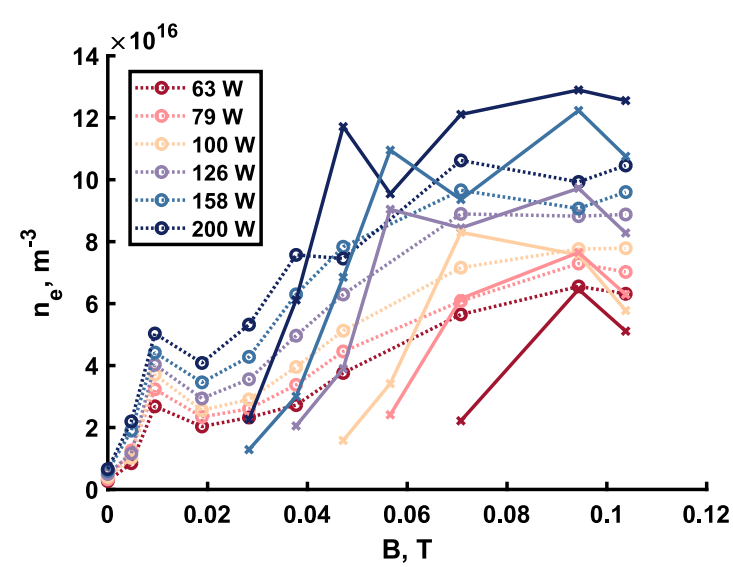

FIG. 3. Densities measured with the Langmuir probe (dotted lines with circle markers) compared to interferometry (solid lines with cross markers) for different power levels. 


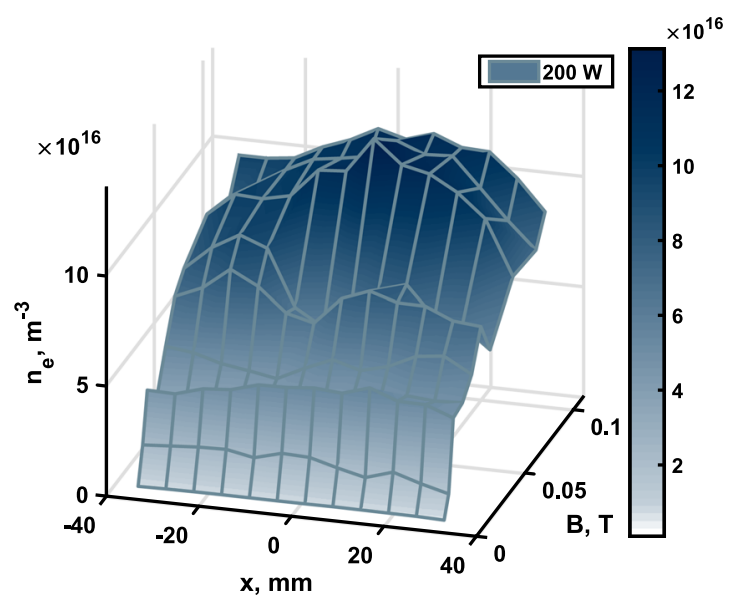

FIG. 4. Spatial density profiles for various B.

The coupled power dependence on B was measured for different forwarded power (Fig. 5). The coupling is generally not affected by the probe presence.

Now a 2D density profile can be constructed as a function of the coupled power and the magnetic field with both probe and interferometry measurements (Fig. 6). The discrepancies

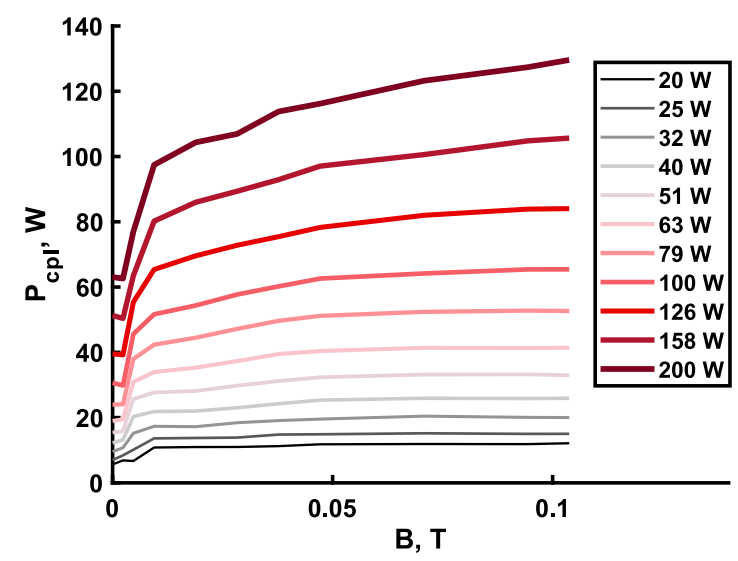

FIG. 5. Variation of the coupled power for various forwarded power.

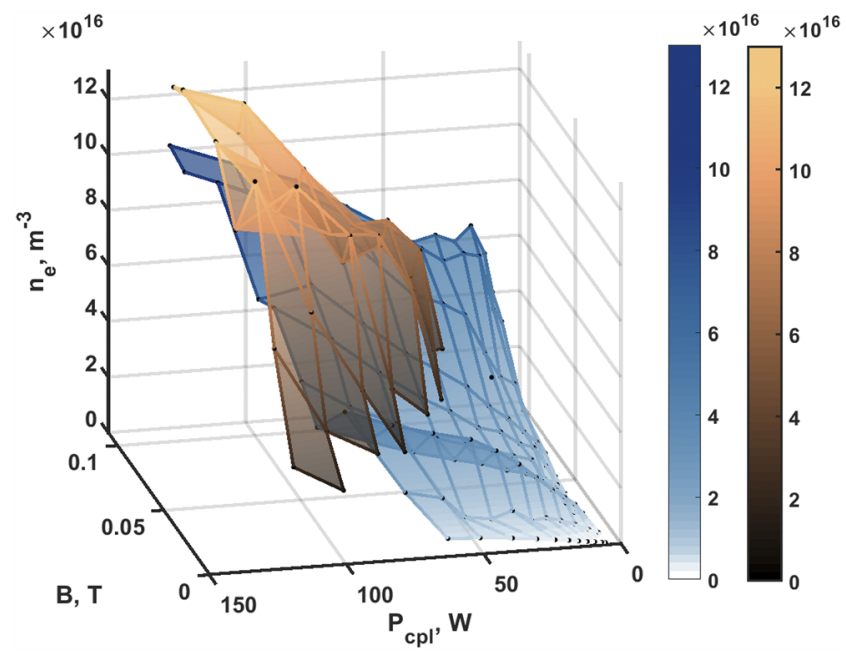

FIG. 6. Density profiles obtained from the interferometry (black to orange color scale) and the Langmuir probe data (white to blue). of the results are mostly within $20 \%$, reaching $50 \%$ for few points of low interferometry signal.

\section{APPLICABILITY ANALYSIS}

An exact formula is undoubtedly preferable to an approximate theory. Equation (13), while being very complex, should be used instead of the inexact effective area formula ${ }^{1}$ when a probe parallel to B is considered. However, construction of a similar analytical procedure for an arbitrary angle of inclination $\theta$ is an unjustifiably sophisticated task. Instead we conduct an analysis of the applicability of the approximate theory. ${ }^{1}$

The simplicity of the resulting formula (17) in the previous work $^{1}$ is achieved by neglecting the fact that the collecting areas depend on a particle speed. Moreover, no distinction for different speeds in the $\mathrm{z}$ direction is drawn. Consequently, the effective area is not an exact expression but an approximation. Limits to its application can be set by revising separately the two terms of Eq. (11). ${ }^{1}$

1. The "non-magnetized" flux $\Gamma_{n-m a g n}$ defined by the condition of the radial speed to be bigger than $v_{\lim r}$ is said to be collected by the whole probe area $S_{p r}$, which is an overestimation. In reality, an electron with $v_{z} \leq v_{\lim z} \cos (\theta)$ strikes a probe no later than one revolution of the cyclotron orbit, $L_{z}=v_{z} * 2 \pi / \omega_{c}$, so all such particles are collected by a smaller area $2 \pi r_{p r} L_{z}+\pi r_{p r}^{2}$. Only particles with $v_{z}$ bigger than $v_{\lim z}$ are collected randomly along the whole probe length. For Eq. (17) to be applicable, they should constitute the majority of the velocity distribution. It is roughly true when the mean thermal velocity $\bar{v}$ is bigger than $v_{\lim z} \cos (\theta)$.

The described overestimation seems to be significant for bigger magnetic fields and smaller $\theta$, when most of the electrons have $v_{z}$ smaller than the limit. However, for bigger magnetic fields, the limitation $v_{r} \geq v_{\lim r}$ leads to a small number of particles in $\Gamma_{n-m a g n}$ since $v_{\lim r}$ is also quite big (depending on the temperature). Quantitatively the conditions for the first remark to be insignificant can be expressed as

$$
\left[\begin{array}{l}
\bar{v}>v_{\lim z} \cos (\theta) \text { for any } r_{p r} \\
\bar{v}<v_{\lim z} \cos (\theta) \text { and } \bar{v}<v_{\lim r}
\end{array} .\right.
$$

2. The second limitation concerns the "magnetized" flux. If a center of the cyclotron rotation of an electron with $v_{r} \leq v_{\lim r}$ lies outside the perpendicular probe projection but at a distance no longer than $r_{c e}$ from it, the electron trajectory intersects the probe and such a particle is collected. However, it may be collected not only by the perpendicular surface as was assumed previously but also by a part of the parallel surface proportional to its speed $v_{z}$. The "magnetized" flux is large only in strong magnetic fields. At the same time, most of the electrons have speeds $v_{z}<v_{\lim z} \cos (\theta)$, so the additional area $2 \pi r_{p r} L_{z}$ is quite small. The second correction is always very small and negligible compared to the effect of the first correction. 
Finally, the criteria of the applicability of the approximate theory are those described by (14). When they are not satisfied, the effective area is overestimated up to the factor of $S_{p r} / S_{\perp}$. A more precise overestimation value can hardly be provided.

We can review the application to the experimental data carried out in the previous work ${ }^{1}$ for two cylindrical probe tips. For the smaller tip of $\mathrm{r}_{\mathrm{pr}}=75 \mu \mathrm{m}, \mathrm{L}_{\mathrm{pr}}=1 \mathrm{~cm}$, Eq. (14) gives that $S_{\text {eff }}$ is acceptable only for $B<0.0076 \mathrm{~T}$ and $B>0.114 \mathrm{~T}$ at $\theta=5^{\circ}$, which means that for nearly the whole range of $\mathrm{B}$ the resulting densities are underestimated. The bigger probe $\left(\mathrm{r}_{\mathrm{pr}}=0.5 \mathrm{~mm}, \mathrm{~L}_{\mathrm{pr}}=1 \mathrm{~cm}\right)$ data gave larger span of reliable results, $B<0.0076 \mathrm{~T}$ and $B>0.017 \mathrm{~T}$, so only a small part in between these two values resulted in overestimated $S_{\text {eff }}$ and underestimated $n$.

We plot Eq. (13) from this paper and (17) from the previous work ${ }^{1}$ for $\theta=0^{\circ}$ (Figs. 7 and 8) to check the results from the paragraph above and thus the validity of the assumptions (14). All curves are for a constant temperature of $5 \mathrm{eV}$. The boundaries of $\mathrm{B}$ are prolonged beyond the ones used in the experiment to see the upper threshold value where the two theories start to match.

The lower threshold values are not observed. The values of the upper threshold in both cases are twice bigger than those obtained from (14). This can serve as a guidance to take the criteria for the approximate theory applicability as those in (14) but with a factor of 2 for $\bar{v}<v_{\lim } r$. After some transformations, they look like

$$
\left[\begin{array}{l}
B<\frac{2 \pi m_{e} \bar{v}}{e L_{p r} \cos (\theta)}, \text { for any } r_{p r} \\
B>\frac{2 \pi m_{e} \bar{v}}{e L_{p r} \cos (\theta)} \text { and } B>2 \frac{m_{e} \bar{v}}{e r_{p r}}
\end{array} .\right.
$$

The second criterion should be quite correct for a broad range of parameters at $\theta=0^{\circ}$ and even more so for nonzero angle since the overestimation of the area reduces with the angle. The first criterion might be applicable for some non-zero angles, but this question requires further investigations.

The comparison above is performed for $\theta=0^{\circ}$, but $\theta$ was around $5^{\circ}$ in the experiments, ${ }^{1}$ so the real overestimation of $S_{\text {eff }}$ is somewhat lower than what we predict for a parallel probe. The experimental data (Fig. 7 of Ref. 1) show that the underestimation of $n$ for the small probe is maximum of the factor of 2 compared to the big probe. One of the reasons for

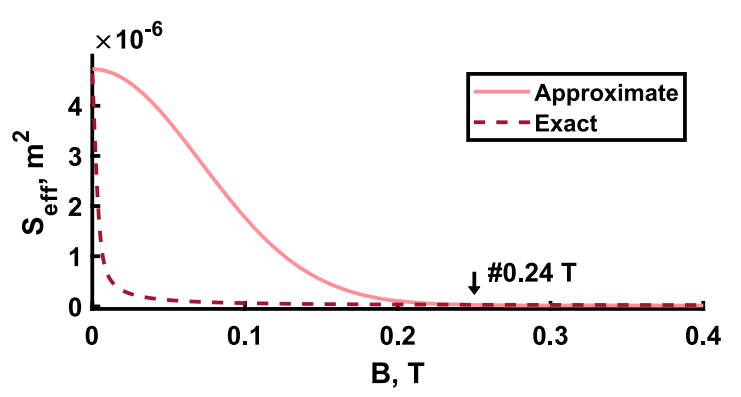

FIG. 7. Comparison of the approximate and exact theories for $\mathrm{r}_{\mathrm{pr}}=75 \mu \mathrm{m}$. The arrow indicates the intersection.

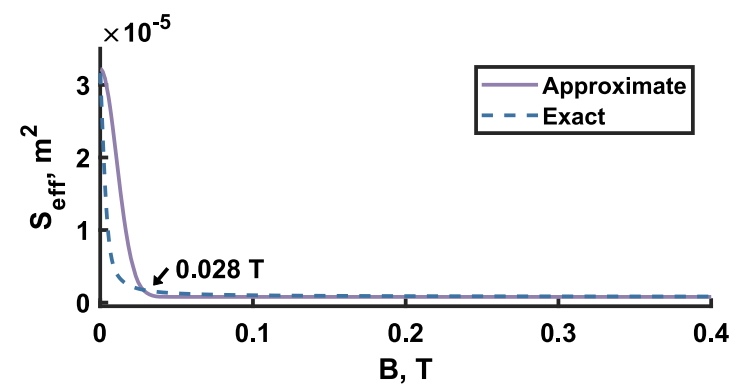

FIG. 8. Comparison of the approximate and exact theories for $\mathrm{r}_{\mathrm{pr}}=0.5 \mathrm{~mm}$. The arrow indicates the intersection.

that is the non-zero angle, but there might be other factors which affected the current collection.

\section{CONCLUSION}

An exact analytical solution is given for the electron current collected at $\mathrm{V}_{\mathrm{pl}}$ by a cylindrical Langmuir probe oriented parallel to the magnetic field. All electrons are divided in 4 fractions according to their radial and parallel to B velocities. The effective collecting area is derived as the sum of the 4 current contributions divided by the electron charge and random thermal flux.

The constructed theory for interpretation of cylindrical Langmuir probe data allowed for the first time to obtain reliable density profiles in magnetized plasma on Aline. Results are obtained for a broad range of the magnetic fields as well as the coupled power levels.

Interferometry measurements of line-integrated densities have been carried out for the same range of parameters. Values that are well above the noise level of $1 \times 10^{16} \mathrm{~m}^{-3}$ match the densities obtained with the probe with up to $20 \%$ error. Possible causes of errors are listed.

The preceding approximate theory ${ }^{1}$ for a cylindrical probe at an arbitrary angle is compared to the exact model for the parallel probe orientation and applicability criteria are formulated. The overestimation of the collecting area by the approximate theory seems big, but the experimental underestimation of density is only of the order of 2 .

\section{ACKNOWLEDGMENTS}

This work has been carried out within the framework of the EUROfusion Consortium and has received funding from the Euratom research and training programme 2014-2018 under Grant Agreement No. 633053. The views and opinions expressed herein do not necessarily reflect those of the European Commission.

\footnotetext{
${ }^{1}$ M. Usoltceva, E. Faudot, S. Devaux, S. Heuraux, J. Ledig, G. V. Zadvitskiy, R. Ochoukov, K. Crombé, and J.-M. Noterdaeme, Phys. Plasmas 25, 063518 (2018).

2 J. G. Laframboise and J. Rubinstein, Phys. Fluids 19, 1900 (1976).

${ }^{3}$ J. Rubinstein and J. G. Laframboise, Phys. Fluids 21, 1655 (1978).

${ }^{4}$ E. Faudot, S. Devaux, J. Moritz, S. Heuraux, P. Molina Cabrera, and F. Brochard, Rev. Sci. Instrum. 86, 063502 (2015).

${ }^{5}$ S. Devaux, E. Faudot, J. Moritz, and S. Heuraux, Nucl. Mater. Energy 12, 908-912 (2017)
} 